\title{
Level and width statistics of the open many-body systems
}

\author{
Shoujirou Mizutori ${ }^{1, a}$ and Hirokazu Aiba ${ }^{2, b}$ \\ ${ }^{1}$ Kansai University of Welfare Sciences \\ ${ }^{2}$ Kyoto Koka Women's College
}

\begin{abstract}
The level and width statistics of the two kinds of the random matrix models coupled to the continuum are analyzed. In the first model, the gaussian orthogonal ensemble with random couplings to the continuum, not only the width statistics deviate from the Porter-Thomas distribution due to the super-radiant mechanism, but also the distribution of the nearest neighbor level spacings shows deviation from the Wigner one simultaneously. In the second model, the two body random ensemble with correlated couplings to the continuum, the correlation between the target and the compound states leads to the global energy dependence of the widths. Within the narrow energy interval where states with widths deviating from the global energy dependence lie, the distributions behave similar way with the case of the random couplings. Namely, the deviation of statistics of the nearest neighbor level spacings from the Wigner distribution and the deviation of the width statistics from the Porter-Thomas distribution take place simultaneously within the models we investigated.
\end{abstract}

\section{Introduction}

The description of the isolated neutron resonances(INR) has close connection with the random matrix theory and the phenomenon of the quantum chaos.

To begin with, N. Bohr's compound nuclear picture[1], which tried to explain the narrow widths of the neutron resonances, implies the chaotic motion of the nucleons in the nuclei. In the 50's, Wigner's random matrix theory(RMT) was proposed as the theory of the INR[2]. The nearest neighbor level spacings(NNLS) following the Wigner surmise, and the widths following the Porter-Thomas(PT) distribution, are well explained by the RMT. In the 80's, the idea of the nuclear data ensemble(NDE) was proposed[3], in which the combination of the data of different nuclei is treated as an ensemble, and regarded as a strong evidence for the RMT. At the same time, it has been established that the RMT is a generic model of the quantum chaos. Hence, there has been no question that the INR are well described by the RMT and are typical examples of the quantum chaos.

However in 2010, it was claimed that the widths of the INR of Th isotopes do not follow the PT distribution with $99.997 \%$ significance[4]. Further claim was raised that the precise re-examination of the NDE indicates the exclusion of the RMT with $98.17 \%$ significance[5]. Although the significance level claimed there might be overestimated[6], these new analysis gave a great doubt on the RMT.

\footnotetext{
ae-mail: mizutori@tamateyama.ac.jp

be-mail: h_aiba@mail.koka.ac.jp
} 
This issue is not restricted to whether a model is correct or not. As the RMT has close relation with the quantum chaos, the problem is concerned with the whole picture of the compound nuclear states. It is natural that the nuclear theory is nudged by the claim[7].

There are several arguments that the distribution of the width may not directly connected with the chaoticity. The expectation that the widths follow the Porter-Thomas distribution arises from the assumption that the randomness of the matrix elements of the intrinsic states reflects directly to the width distribution. There are however several possibilities giving rise to the deviation. The penetration factor may have a modified energy dependence [8], the neutron width may be different from the simple square of the coupling to the continuum due the super-radiant mechanism[9], and/or the randomness of the matrix elements of the intrinsic states may not manifest in the couplings to the continuum because of the correlation between the compound states and the target plus one neutron states[10].

Super-radiance is a phenomenon which occurs in the limit of the strong coupling to the continuum. In such a situation, only a few, the same number with the open channels, states monopolize the coupling to the continuum, and the remaining states have only small widths. If the strength is modestly large, the collectivization is weak and some states have relatively large widths than the others, which may cause the deviation from the PT distribution. Although the super-radiant mechanism together with the correlation between the compound and the target nuclei may explain the deviation of the width statistics from the PT distribution, the effect of the same mechanism on the level statistics has not been investigated so far. Since the level interaction occurs in the complex plane, there must be some effect on the level statistics as is seen in a past result[11]. In this talk, adopting the same model with ref.[9] and ref.[10], we investigate the relation of the width statistics and the level statistics.

\section{The model}

The energies and the widths of the neutron resonances are given[12] as the eigenvalues

$$
\mathscr{E}_{f}=\mathcal{E}_{f}-i \frac{1}{2} \Gamma_{f}
$$

of the effective Hamiltonian

$$
\mathcal{H}=H-i \sum_{c} \mathscr{P}^{c} W_{c}^{T} \cdot W_{c}
$$

where the real part $H$ is the Hamiltonian of the compound nuclei with bound state approximation, and $W_{c}$ stands for the coupling between the bound state and the channel vector associate with the channel c. $\mathscr{P}^{c}$ is the strength of the continuum coupling for the channel c.

We investigate two models. In one model, we adopt the Gaussian Orthogonal Ensemble (GOE) as $H$ and random numbers with normal distribution as each elements of $W_{c}$ [9]. We call this model GOE + random coupling (GOE+RC) model. In the other model, we adopt the two-body random ensemble (TBRE) as $H$ and the values of the overlaps between the compound states and the target plus oneparticle states as the elements of $W_{c}$ [10]. This we call TBRE + correlated coupling (TBRE+CC) model. In both cases, the discussion is restricted in the case of single channel. So we omit the subscript $c$ hereafter.

The deviations from the GOE limit, namely the Wigner surmise in the case of NNLS and the PT distribution in the case of widths, are characterlized in terms of the parameter $\beta$ of the Brody 
distribution and the degree of freedom $v$ of the $\chi^{2}$ distribution, respectively. Here,

$$
\begin{gathered}
P_{B}(S)=a S^{\beta} \exp \left(-b S^{\beta+1}\right), \\
a=(\beta+1) b, \\
b=\left\{\Gamma\left(\frac{\beta+2}{\beta+1}\right)\right\}^{\beta+1},
\end{gathered}
$$

is the Brody distribution and

$$
f(x ; v,\langle x\rangle)=\frac{(1 / 2)^{v / 2}}{\Gamma(v / 2)}\left(\frac{x}{\langle x\rangle}\right)^{v / 2-1} \exp \left(\frac{-v x}{2\langle x\rangle}\right)
$$

is the $\chi^{2}$ distribution.

\subsection{GOE+random coupling model}

We first check the GOE+RC model. Here, $H$ is composed of the random numbers. Namely,

$$
H_{i j} \in N\left(0,1+\delta_{i j}\right)
$$

where $N(r, s)$ is the normal distribution with average $r$ and variance $s$. $W$ is also composed of the random numbers,

$$
W_{i} \in N(0,1)
$$

The coupling constant $\mathscr{P}$ is scaled as

$$
\tilde{\kappa}=\mathscr{P} / \sqrt{\Omega}
$$

where $\Omega$ is the dimension of the random matrix.

\subsection{TBRE+correlated coupling model}

The basic ingredients of the TBRE are the two body interactions,

$$
\begin{aligned}
V= & \sum_{i>j, k>n} v_{i j k n} c_{i}^{\dagger} c_{j}^{\dagger} c_{n} c_{k} \\
& v_{i j k n} \in N\left(0,1+\delta_{i k} \delta_{j n}\right)
\end{aligned}
$$

where $c_{i}^{\dagger}$ is the creation operator of the single particle. The number of levels is expressed as $l$.

The basis states with particle number $m$ are constructed as

$$
|\alpha ; m\rangle=\Pi_{n(\alpha)=1}^{m} c_{i_{n(\alpha)}}^{\dagger}|0\rangle
$$

where $\left\{i_{n(\alpha)}\right\}$ indicate a set of $m$ single particle labels, and the basis states with particle number $d=$ $(m-1)$ are constructed as

$$
|\mu ; d\rangle=\Pi_{n(\mu)=1}^{d} c_{i_{n(\mu)}}^{\dagger}|0\rangle .
$$

The matrix elements of the real part of the Hamiltonian are

$$
H(m)_{\alpha \beta}=\left\langle\alpha ; m\left|\sum_{i>j, k>n} v_{i j, k n} c_{i}^{\dagger} c_{j}^{\dagger} c_{n} c_{k}\right| \beta ; m\right\rangle,
$$


while those of the target states are

$$
H(d)_{\mu \nu}=\left\langle\mu ; d\left|\sum_{i>j, k>n} v_{i j, k n} c_{i}^{\dagger} c_{j}^{\dagger} c_{n} c_{k}\right| v ; d\right\rangle .
$$

The coupling with continuum is assumed to occur through the channel vector state $|\mathscr{C} ; m\rangle$. We choose as this state, in the TBRE+CC model, adding a single particle to the target ground state, $\left|\sigma_{0} ; d\right\rangle$. To define the single-particle, we make use of the eigenstates of the density matrix,

$$
\rho_{i j}=\delta_{i j}-\left\langle\sigma_{0} ; d\left|c_{i} c_{j}^{\dagger}\right| \sigma_{0} ; d\right\rangle
$$

Namely we compose a creation operator $c_{a}^{\dagger}$ as

$$
\begin{gathered}
c_{a}^{\dagger}=\sum_{i} f_{a i} c_{i}^{\dagger} \\
\sum_{j} \rho_{i j} f_{a j}=n_{a} f_{a i} .
\end{gathered}
$$

Then the channel vector is constructed as

$$
|\mathscr{C} ; m\rangle=\left|a+\sigma_{0} ; m\right\rangle \equiv \mathcal{N}\left\{c_{a}^{\dagger}\left|\sigma_{0} ; d\right\rangle\right\}
$$

where $\mathcal{N}$ indicates taking normalization. Another assumption is that the compound states with energy lower then the target ground state do not couple with the continuum.

With these two assumptions, the coupling matrix elements to the continuum in the basis of the eigenstate of the compound Hamiltonian are expressed as

$$
\begin{array}{cc}
\left.W_{\lambda}=\left\langle a+\sigma_{0} ; m \mid \lambda ; m\right\rangle\right\rangle & \text { for } E_{\lambda}>0 \\
=0 & \text { for } E_{\lambda}<0
\end{array}
$$

where $E_{\lambda}$ is the energy of the compound state $\lambda$ relative to the target ground state.

The coupling strength with the continuum is controlled in terms of the parameter $\kappa$, defined as

$$
\kappa=\mathscr{P} / \lambda,
$$

where $\lambda^{2}=\frac{1}{\Omega} \operatorname{Tr}\left(H^{2}\right)$ and $\Omega$ is the size of the Hamiltonian $H(m)$.

\section{Results}

\subsection{GOE+RC model}

Figure 1 shows the deviation from the GOE limit when the coupling to the continuum increases. The filled squares show the parameter $\beta$ in eq.(3) while the filled circles are for the degrees of freedom $v$ of the $\chi^{2}$ distribution in eq.(4). As is shown, when $\tilde{\kappa}$ is increased, both $\beta$ and $v$ decrease, untile $\tilde{\kappa}$ becomes around 1 . With increasing $\tilde{\kappa}$ further, both $\beta$ and $v$ become close to unity.

To show the reason of this behavior, we plotted the positions of the eigenvalues in the $\mathcal{E}-\Gamma$ plane for $\tilde{\kappa}=0.1$ (panel a), 1.0(panel b), 10.0(panel c) in fig. 2. As is seen in panel a), the widths do not have any energy dependence for small $\tilde{\kappa}$. For $\tilde{\kappa}=1.0$ we see some widths relatively large but not isolated from others around $\mathcal{E}=0$ in panel b). In this situation, these states with large widths may have close real energies to other states with small widths, since the energy repulsion takes place in the complex 

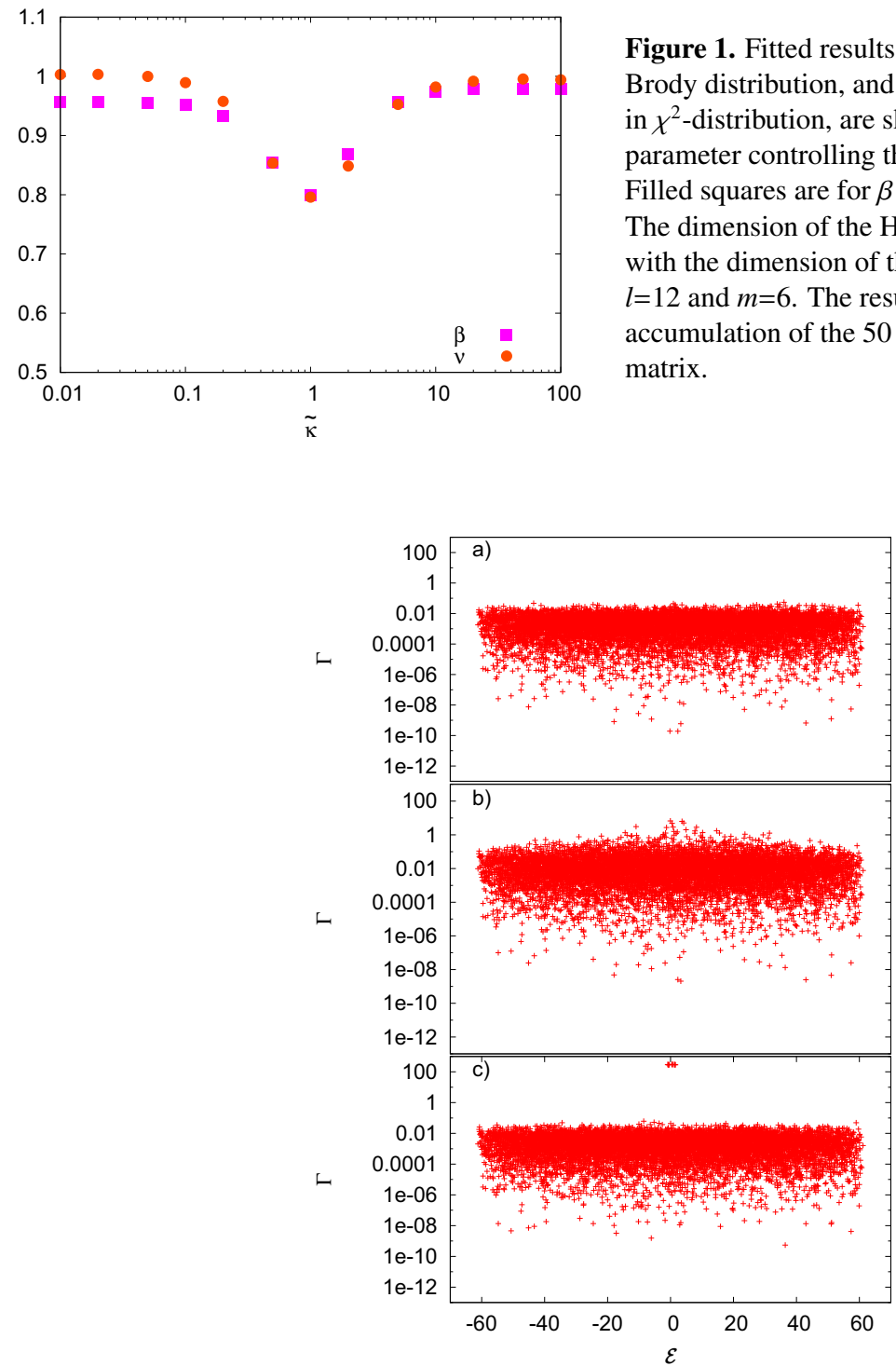

Figure 2. The positions of the complex eigenvalues in the $\mathcal{E}-\Gamma$ plane for $\tilde{\kappa}=0.1$ (a), 1.0 (b), 10.0 (c). The dimension of the Hamiltonian $=924$ and the plots are accumulated results of 10 realizations of the random matrix.

plane. Therefore, the reason which drive to the deviation of the width distribution from the PT one causes also the deviation of the NNLS distribution from Wigner one, as states with large difference in width(the imaginary axis) may have small level distance(the real axis). With further increase of $\tilde{\kappa}$ to 10.0 , as is seen in panel c), states with large widths (one state for each realization) are separated from the other states and have no significance in the statistics, while the widths of the other states follow PT distribution as they are created by small, random real perturbations to a state with large width. 

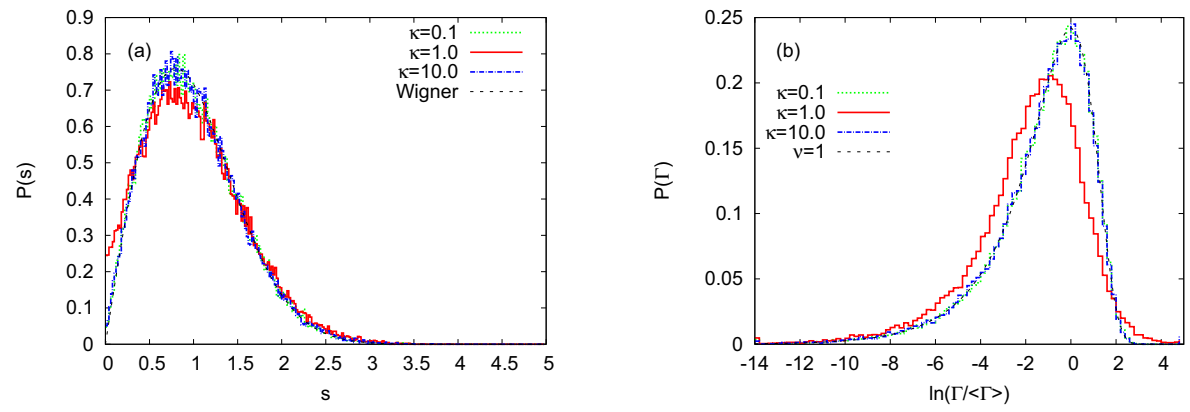

Figure 3. The NNLS distribution (a) and the width distribution(b) obtained from GOE+RC model for $\tilde{\kappa}=$ 0.1(dotted line), $\tilde{\kappa}=1.0$ (solid line), $\tilde{\kappa}=10.0$ (dashed line) compared to the Wigner one (a) or Porter-Thomas one(b). $\Omega=924$, and the number of realizations is 50. For $\tilde{\kappa}=0.1$ and $\tilde{\kappa}=10.0$ the results are close to the Wigner one or PT one while for $\tilde{\kappa}=1.0$ the results are different.

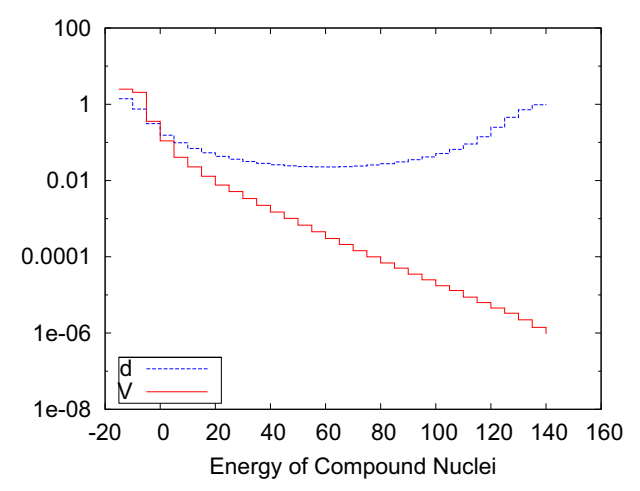

Figure 4. The $E_{\lambda}$ dependence of the size of the coupling to the continuum $V_{\lambda}=W_{\lambda}^{2}$, compared with the level spacing $d$. The values are the results of the averaging over an energy interval $\Delta_{E}=5$ and 50 realizations. In this figure, the values for $E_{\lambda}<$, which are not included in the effective Hamiltonian (2), are also plotted.

In fig. 3, the NNLS distribution (a) and the width distribution(b) are compared for $\tilde{\kappa}=$ $0.1,1.0,10.0$ together with the simple GOE prediction, namely the Wigner one (a) or the PT one(b). They are the combined plots for 10 realizations of the random matrix. In both $\tilde{\kappa}=0.1$ case and $\tilde{\kappa}=10.0$ case, the distributions are very close to the GOE ones, while for $\tilde{\kappa}=1.0$ there are significant differences.

\subsection{TBRE + correlated coupling model}

The most significant difference of the TBRE+CC model from the GOE+RC model is the strong $E_{\lambda}$ dependence of the coupling $W_{\lambda}$. In fig. 4 , the average of the coupling strength $W_{\lambda}^{2}$ over an interval $\Delta_{E}$ and realizations is plotted as a function of $E_{\lambda}$. For small $\kappa$, the coupling can be treated perturbatively, hence the widths have the similar dependence on $\mathcal{E}_{f}$.

In fig. 5, positions of the complex eigenvalues are plotted on the $\mathcal{E}-\Gamma$ plane for the TBRE+CC model with $l=14$ and $m=7$ and $\kappa=0.1$ (panel a), 1.0(panel b) and $10.0($ panel $\mathrm{c})$. They are the combined plots for 10 realizations of the random matrix. Contrary to the case of the GOE+RC model, seen in fig 2 , the widths have overall energy dependence in all cases. 


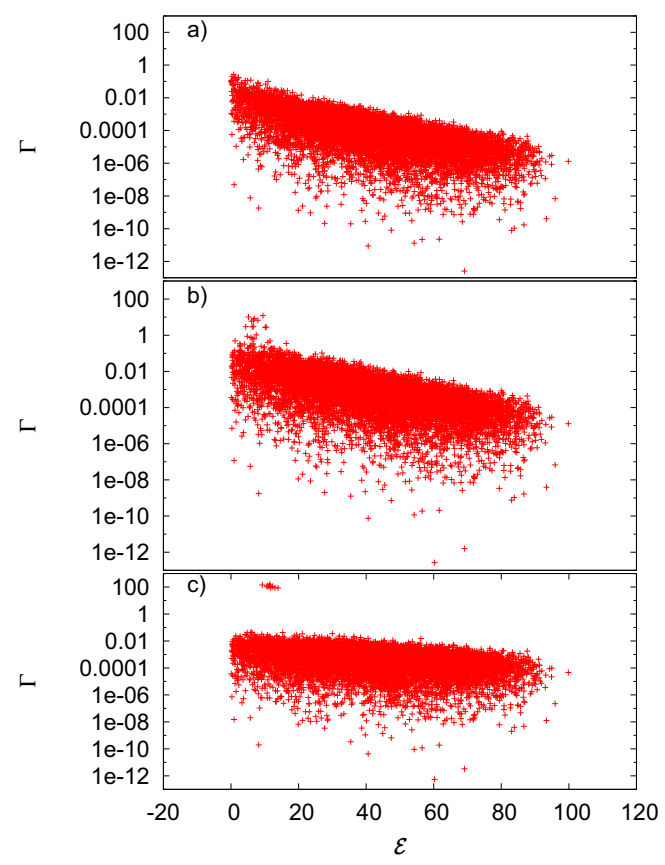

Figure 5. The positions of the complex eigenvalues in the $\mathcal{E}-\Gamma$ plane for $\kappa=0.1$ (a), 1.0 (b), 10.0 (c). The Hamiltonian is for $l=14$ and $m=7$, and the plots are accumulated results of 10 realizations.

Another difference is that the energy interval where the states with large widths deviating from the global energy dependence for $\kappa \simeq 1$ exist is much narrower in the case of the TBRE+CC model. (Compare the panel (b) of figs. 2 and 5.)

This strong dependence of average $\Gamma$ on $\mathcal{E}_{f}$ derives a completely different distribution of $\Gamma$, as is seen in [10], if the states all over the energy region are considered. However, in the real situation of INR, neutron widths are analyzed within very small intervals compared with the whole energy region of the excited states of nuclei. Therefore, we restricted our analysis within a small energy interval in which the effect of the global energy dependence of the average width is, if not negligible, small.

In fig. 6, the NNLS distribution (a) and the width distribution(b) obtained from the eigenvalues in the interval $5.0 \leq \mathcal{E} \leq 25.0$ are compared for $\kappa=0.1,1.0,10.0$ together with the Wigner one(a) or PT one(b). Both $\kappa=0.1$ case and $\kappa=10.0$ case, the distributions are very close to the Wigner one (a) or PT one, while for $\kappa=1.0$ there are significant difference.

In fig. 7, we plot $\beta$ and $v$ obtained from the TBRE+CC model with $l=14, m=7$. The analysis is done in the energy interval $5 \leq \mathcal{E} \leq 20$. Although less clear than in the result of the GOE+RC model, the deviation from the unity is seen in the intermediate region of coupling strength around $\kappa \simeq 1$.

Such deviation is seen only in the limited energy region. In fig. 8, the dependence of $v$ on $\kappa$ in the different energy interval is compared. Unlike the interval $5 \leq \mathcal{E} \leq 20$, the behavior in the interval $20 \leq \mathcal{E} \leq 35$ is less significant, and is invisible in the interval $35 \leq \mathcal{E} \leq 50$. 

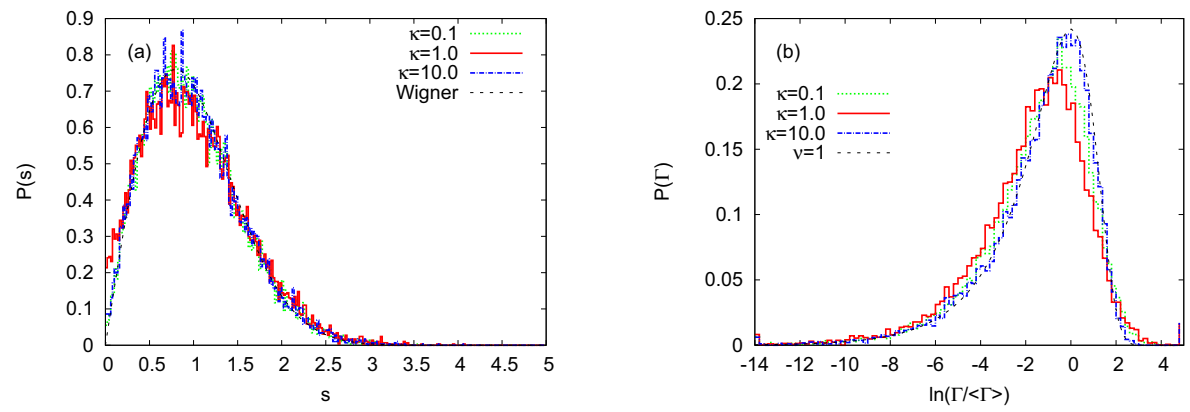

Figure 6. The NNLS distribution (a) and the width distribution(b) obtained from the TBRE+CC model in the energy interval $5.0 \leq \mathcal{E} \leq 25.0$ for $\kappa=0.1$ (dotted line), $\kappa=1.0$ (solid line), $\kappa=10.0$ (dot-dashed line) compared to the Wigner one (dashed line) (a) or PT one (b). $l=14$ and $m=7$, and the number of realizations is 50. For $\kappa=0.1$ and $\kappa=10.0$ the results are close to the Wigner one or the PT one while for $\kappa=1.0$ the results are different.
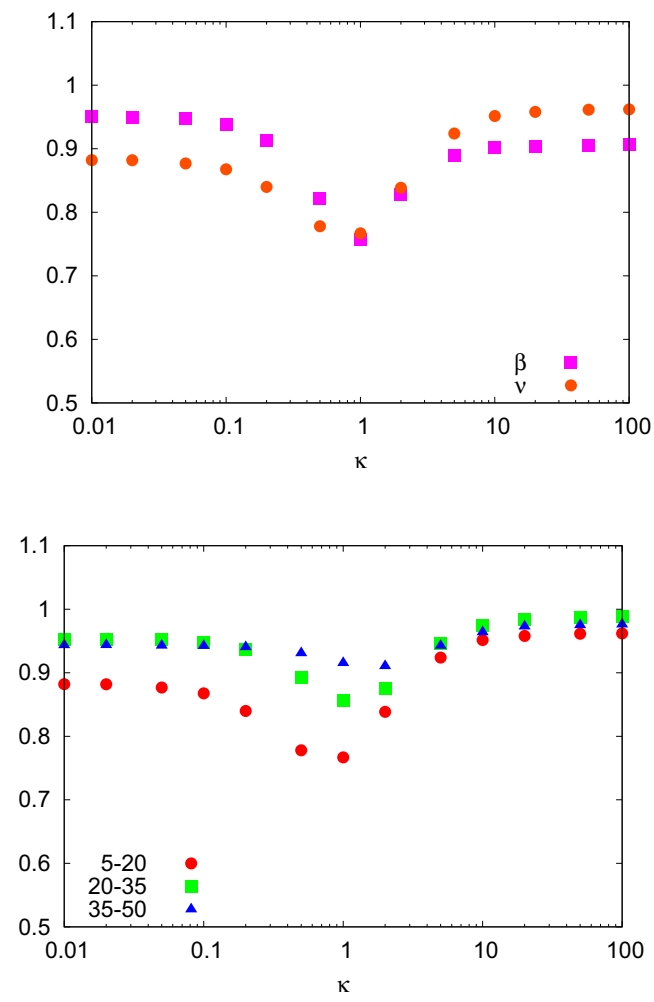

Figure 7. $\beta$ (square) and $v$ (circle) obtained from the result of the TBRE+CC model with $l=14, m=7$. States in the energy interval $5 \leq \mathcal{E} \leq 20$ are considered. The number of realizations is 50 .

Figure 8. $v$ obtained from the TBRE $+\mathrm{CC}$ model with $l=14, m=7$. The circles are the result s obtained in the energy interval $5 \leq \mathcal{E} \leq 20$ (same with the ones in fig. 7 ), while the pluses (the crosses) are the ones in the interval $20 \leq \mathcal{E} \leq 35(35 \leq \mathcal{E} \leq 50)$, respectively. The number of realizations is 50 . 


\section{Conclusion}

Motivated by the recent claim that the neutron resonance width distribution does not support the random matrix theory, we examine the consequence of the super-radiant mechanism in the strong continuum coupling and the effect of the correlation between the target and the compound nuclei on the statistics of both the levels and the widths simultaneously, by controlling the strength to the continuum.

If the coupling to the continuum is large, several states have relatively large widths, resulting the deviation from the GOE limit both in the nearest neighbor level spacing distribution and the width distribution. However in the limit of strong coupling, the behaviors of both levels and widths are restored to the GOE ones, because only one state monopolizes the coupling to the continuum in this limit, and the rest states have the normal statistical property.

The effect of the correlation between the target and the compound states results in the global excitation energy dependence of the widths. Therefore, the width distribution for whole spectrum is completely different from the GOE one. However, if the width distribution is evaluated within a relatively narrow energy interval, it approximately follows the Porter-Thomas distribution as long as the coupling to the continuum is week.

When the coupling to the continuum becomes large, both the NNLS distribution and the width distribution deviate from the ones of the GOE in the energy interval where the states with large widths crowd. However, in other energy intervals away from "width concentrating region", the distributions are similar to the GOE ones. With further increase of the strength, both the NNLS distribution and the width distribution come again close to the GOE ones.

The deviation of the width distribution from the Porter-Thomas one and the deviation of the NNLS distribution from the Wigner one occur coincidently within the model we adopted.

\section{References}

[1] N. Bohr, Science 137, 344 (1936)

[2] C.E. Porter, Statistical Theories of Spectra: Fluctuations (Academic, New York, 1965)

[3] R. U. Haq, A. Pandey, and O. Bohigas, Phys. Rev. Lett. 48, 1086 (1982)

O. Bohigas, R. U. Haq, and A. Pandey, Nuclear Data for Science and Technology (Reidel, Dordrecht, 1983) 809

[4] P. E. Koehler et al, Phys. Rev. Lett. 105, 072592 (2010)

[5] P. E. Koehler, Phys.Rev. C 84, 034312 (2011)

[6] J. F. Shriner Jr, H. A. Weidenmüller, and G. E. Mitchell, arXiv:1209.2439v3 (2014)

[7] E. G. Reich, Nature, 466, 1032 (2010)

[8] H.A. Weidenmüller, Phys. Rev. Lett. 105, 232501(2010)

[9] G. L. Celardo, N. Auerbach, F. M. Izrailev, and V. G. Zelevinsky, Phys. Rev. Lett. 106, 042501(2011)

[10] A. Volya, Phys. Rev. C 83, 044312(2011)

[11] S. Mizutori and V. G. Zelevinsky, Zeit. für Phys. A 346, 1(1993)

[12] H. Feshbach, Ann. Phys. (NY) 5, 357(1958)

C. Mahaux and H. A. Weidenmüller, Shell-model approach to nuclear reactions (North Holland, Amsterdam, 1969) 